\title{
Price Coordination in Distribution Channels: A Dynamic Perspective ${ }^{\text {kT }}$
}

\author{
Guiomar Martín-Herrán ${ }^{1, *}$, Sihem Taboubi ${ }^{2}$
}

\begin{abstract}
In this study, we investigate two important questions related to dynamic pricing in distribution channels: (i) Are coordinated pricing decisions efficient in a context where prices have carry-over effects on demand? (ii) Should firms practice a skimming or a penetration strategy if they choose to coordinate or to decentralize their activities? To answer these questions, we consider a differential game that takes place in a bilateral monopoly where the past retail prices paid by consumers contribute to the building of a reference price. The latter is used by consumers as a benchmark to evaluate the value of the product, and by firms to decide whether to adopt a skimming or a penetration strategy.

We then compute and compare strategies, total channel profits and individual profits under vertical integration and decentralization at steady state and along the optimal time-paths. One of our main findings states that, for some values of the initial reference price, there is a time interval where channel decentralization performs better than coordination. During this transition period, at least one of the channel members could be tempted to end his cooperation, especially if he is not farsighted and if there are no binding agreements with the other channel partners.
\end{abstract}

Keywords: Marketing channels, dynamic pricing, channel coordination, differential games.

\section{Introduction}

Many studies have investigated the issue of pricing in marketing channels by taking into account the interactions between their different members and their impact on

\footnotetext{
The authors wish to thank Georges Zaccour and three anonymous reviewers for their valuable comments and suggestions. The first author's research is partially supported by MICINN under projects ECO2008-01551/ECON, ECO2011-24352, co-financed by FEDER funds and the COST Action IS1104 "The EU in the new economic complex geography: models, tools and policy evaluation". The second author's research is supported by NSERC, Canada.

*Departamento de Economía Aplicada (Matemáticas), Universidad de Valladolid, Avda. Valle de Esgueva, 6, 47011, Valladolid, Spain. Tel.: +34 983423330 Fax: +34 983 423299; E-mail: guiomar@eco.uva.es

${ }^{1}$ IMUVA, Universidad de Valladolid, Spain, and GERAD, Montréal, Canada.

${ }^{2}$ GERAD and Marketing Department, HEC Montréal, Canada.
} 
channel members strategies and profits. The seminal work of Jeuland and Shugan (1983) was one of the first studies to demonstrate the benefits of vertical integration (i.e. cooperation or coordination) with respect to channel decentralization for channel members and consumers. This study triggered a subsequent work in the field of gametheoretic modelling in distribution channels. With some exceptions, most of these studies examined the interactions between channel members in a static environment. By doing so, they ignored the fact that some marketing variables also have long-term effects that could be damaging to demand in the long run. Promotional activities are an example of such variables. Despite the increase in short-run sales after pricepromotional activities, their long-term impact is not always positive, as "frequent price reductions and deals may serve to increase consumer resistance to choosing a brand when it is sold at a regular price" as reported in Krishnamurthi et al. (1992) (see also Kalyanaram and Winer (1995). This seriously brings into question whether some of the results on the efficiency of static pricing decisions in coordinated distribution channels still hold if we adopt a dynamic perspective when examining this issue.

Indeed, the immediate impact of price on demand is negative and is captured in most of the traditional economic-theory models used in channel literature. When we introduce into these models the carry-over impact of pricing strategies, we take into account the fact that the past prices paid by consumers contribute to the building of a reference price. The latter is compared to the actual retail price, which leads to a judgment about the observed retail price (cheap or expensive) and a perception of the value of the product (gain or loss) that affects consumers purchase decisions. Hence, the low retail prices associated to channel coordination in the static setting are not necessarily an optimal solution if we consider their long-term effects because they result in lower reference prices and consequently enhance consumers' perception of a "loss" when they have to pay for the product at the regular price (Nicolau, 2013).

This means that firms should make pricing decisions based not only on the immediate (negative) impact of prices on demand, but also on their long-term impact. ${ }^{3}$ This will also dictate whether firms should practice a skimming or a penetration strategy by pricing the products above or below the consumers' reference price (Völckner, 2008). The importance of considering both the immediate and long-term effects of prices increases in a channel's context where channel members pricing decisions and profits are interdependent. Indeed, the efficiency of vertical integration, as suggested in the static channels literature, should be reexamined along the whole planning horizon, as one of its implications is a decrease of retail prices, and consequently, a decrease of reference prices and an increase of consumers' perception of a price surcharge when the retail

\footnotetext{
${ }^{3}$ According to Popescu and $\mathrm{Wu}$ (2007), managers who ignore the long-term effects of their pricing strategies can lose revenues. Nasiry and Popescu (2011) provide the example of Apple with its iTune store to illustrate the importance of considering the carry-over effects of prices in pricing strategy. Apple used to sell digital songs for a low price, which, for a long time, was set at 99 cents. When the company decided to raise its prices to $\$ 1.29$, the drop in sales was higher than expected. These lost sales are explained by the fact that the 99 cents that consumers used to pay for these songs became a reference price in their minds, and the new price was perceived as very high compared to this new benchmark.
} 
price is compared with a low reference price.

Zaccour (2006) criticized the static models used in the game-theoretic channels' literature for not being able to "capture the whole picture". Taking into account the dynamic aspects of the market is not only a way to introduce a more realistic feature into these models but it is also a managerial requirement to make strategic decisions. According to Weisstein et al. (2013), dynamic pricing is becoming a prevalent practice in the marketplace, especially in the online retailing, where advances in technology are helping retailers to adjust their prices almost instantaneously.

By considering a dynamic perspective in the study of distribution channels, we take into account the fact that channel members do not interact only once, but could develop or put an end to their partnership during the planning horizon depending on whether there are incentives or barriers to channel coordination. Hence, we allow channel members to deviate from their initial behavior at any point in time. This situation could occur in real-life situations, especially if the different channel members do not act in a farsighted manner or have any binding agreement with the other partners.

In this paper, we use the paradigm of differential games to investigate the issue of pricing when we bring in its dynamic effects on consumer behavior in the channels' literature. We consider a manufacturer and a retailer (i.e. a bilateral monopoly) that fix the transfer and the retail prices respectively, and consider that the evolution of retail prices contributes to the building of a reference price. We study a two-player differential game with one state variable (the reference price) and analyze two scenarios depending on the channel structure. In a first scenario (channel decentralization) each channel member (manufacturer/retailer) has one control variable (transfer/retail price). In a second scenario (vertical integration), the unique decision maker (the channel owner) has a unique control variable (the retail price). We compute channel members' strategies and profits at the steady state and along the optimal time-paths under the scenarios of vertical integration and channel decentralization. By comparing the results, we provide answers to the following questions:

- What pricing strategies should firms adopt under vertical integration and decentralization when their decisions have long-term effects?

- Are coordinated pricing strategies always more efficient than uncoordinated strategies?

- Which dynamic pricing strategy (among skimming and penetration) should firms adopt?

A firm setting its prices above consumers' reference price then reducing this price as time goes by practices a skimming strategy. The opposite situation corresponds to a price penetration strategy. In this case, the firm fixes a low retail price when the product is introduced in the market in order to capture a larger market base and increases its price later on (Dean, 1969). The practice of one of these dynamic pricing strategies has a direct impact on consumers' perception of gains or losses and on firm's profits during the planning horizon. Indeed, a firm practicing a skimming (penetration) strategy has a 
reduced (higher) demand at the introductory stage, but its high (low) prices contribute to the building of a higher (lower) reference price, which allows consumers to perceive a gain (loss) when prices stabilize at the steady state, resulting in a higher (lower) demand at that stage.

Hence, the adoption of a particular dynamic pricing strategy by firms should be affected not only by the initial reference price in consumers' minds, but also by the channel's structure as the latter has a direct impact on the product's retail prices. One of the questions addressed in this paper investigates which strategy among skimming or penetration should firms adopt whether they choose to coordinate or not their decisions (i.e. under vertical integration versus decentralization).

Our paper is structured in the following manner. In Section 2, we provide a brief literature review on the issue of pricing in marketing channels. In Section 3, we present the dynamic model to study this topic. Section 4 gives the main analytical results and the numerical simulations, and Section 5 provides the conclusion.

\section{Literature review}

The impact of the distribution channel's structure on channel members pricing decisions and profits is a topic that has interested many researchers in the areas of marketing and operations research. One of the key findings in this literature states that the highest channel's profit is reached when the channel members coordinate their decisions as if the channel was vertically integrated (Jeuland and Shugan, 1983). This efficiency is explained by the absence of double marginalization (Spengler, 1950). Indeed, the profit margin of a vertically integrated channel is lower than the sum of the individual margins fixed by the decentralized institutions, resulting in lower retail prices, higher demand, and a higher consumer surplus (Jeuland and Shugan, 1983).

These findings were examined later on by different authors who tested if they still hold under different pricing schemes and competitive channel structures (See Ingene et al. 2012). However, most of this literature is based on static models that ignored the carry-over effects of prices on channel members strategies and profits. These carryover effects have been considered in the behavioral pricing literature (Monroe, 1979; Dodds et al. 1991) which relies on the Adaption Level theory (Helson, 1964) to explain how consumers react to price information. According to this theory, the past retail prices observed by consumers in their shopping experiences create adaptation levels, called internal reference prices and consumers' purchase decisions are determined by the discrepancy between the current levels of prices and the reference prices (Monroe, 1979; Fibich et al., 2003). The Transaction Utility theory (Thaler, 1985) provides an additional perspective on the behavioral reaction of consumers to price information. It states that consumers do some mental accounting when they compare the retail prices of products to their internal reference prices. These comparisons result in a perceived sacrifice or loss (deal, or gain) if they buy the product at a retail price that is higher (lower) than their reference price but also a perceived quality effect because of the inferred link between price and quality. The tradeoff between both effects affects their evaluations of the product value and their willingness to buy the product. 
Hence, the long-term price effects may contribute to additional sales if the retail price is lower than the reference price, or to lost sales in the opposite situation. Furthermore, as the variations in retail price affect the reference price, frequent price reductions (increases) might result in a low (high) reference price, which could negatively affect the perception of gain (loss). Consequently, from the firms' perspective, knowledge of the reference price and its effect on the buying behavior of consumers should have an impact on the choice among coordination (i.e. vertical integration) or decentralization of channel members pricing decisions.

Furthermore, the information about reference prices allows firms to choose between a skimming or a penetration pricing strategy. When they choose to follow a penetration strategy, firms agree to sell the product at a relatively low retail price (with respect to the reference price), and then increase the retail price to reach the reference price. This leads to a situation where they benefit initially from additional sales due to the gain perceived by consumers, but this benefit is then reduced when the retail price reaches the reference price ${ }^{4}$ and a perceived loss replaces the perceived gains. The opposite case corresponds to a skimming strategy, where the retail price is fixed to a higher level compared with the reference price to skim the market at the early stages of product introduction (Dean, 1969). Both strategies have an impact not only on retailer's sales and profits, but also on manufacturer's outcome, depending on whether the channel is vertically integrated or decentralized.

To the best of our knowledge, this paper is one of the first to attempt to examine the channel coordination issue by taking into account the dynamic effects of pricing decisions via the reference price dynamics. Previous studies examined the dynamic pricing strategies of firms by focusing either on a unique decision maker (Popescu and Wu, 2007; Nasiry and Popescu, 2011 ) or two competing retailers (Kopalle et al., 1996; Fibich et al., 2003). These studies investigate the conditions under which constant or cyclical pricing strategies should be practiced by firms (i.e. either every day low prices or high-low pricing strategies).

Benchekroun et al. (2009) and Martín-Herrán et al. (2012) also examined the dynamic prices in distribution channels; however, their focus was on the impact of myopia $^{5}$ on channel members' strategies and profits. None of these studies addressed the issue of channel coordination in this context.

The extension of the channel-coordination results from a static to a dynamic setting was suggested by Jeuland and Shugan (1983) as an avenue of research following their paper. Zaccour (2006) was one of the first to address this issue. The author considered a bilateral monopoly where the manufacturer controls the wholesale price and the advertising investments. The retailer fixes the retail price and the promotional activities. Dynamics are introduced via an advertising goodwill model (Nerlove and Arrow, 1962) capturing the impact of non-price marketing activities (advertising and promotions) on the building of brand equity.

\footnotetext{
${ }^{4}$ Remember that an increase of the retail price contributes to an increase of the reference price.

${ }^{5} \mathrm{~A}$ channel member is myopic when he ignores the evolution of retail prices when actual and past retail prices affect consumers' purchasing decisions.
} 
Our study is along the same lines as Zaccour (2006) but with one major difference. In our model, we introduce the dynamics through the carry-over effect of retail prices rather than through the non-price variables suggested by Zaccour (2006). By doing so, we are able to concentrate exclusively on the immediate and long-term impact of the same decision variable (i.e., the retail price) without introducing any bias due to the effects of other marketing variables. ${ }^{6}$

\section{The model}

To investigate the impact of the price dynamic effect on pricing decisions in bilateral monopolies, we study a differential game with two players: A unique manufacturer $(\mathrm{M})$ and a monopolist retailer $(\mathrm{R})$. The manufacturer controls his transfer price $w(t)$ and the retailer controls the retail price $p(t)$. We compare two scenarios: one of channel decentralization and another of vertical integration. Under the decentralized scenario, we consider a Stackelberg game where the manufacturer is the channel leader, whereas the retailer accepts the role of follower, as is commonly assumed in the literature.

The carry-over effects of retail prices are captured in the reference price dynamics $R(t)$. As retail prices evolve in the marketplace, ${ }^{7}$ reference prices are continuously updated to take new retail price information into account. We follow Sorger (1988) and Kopalle and Winner (1996) by modelling the reference price evolution as an exponentially weighted average of past prices. The latter could include observed prices for the same brand of this product, those of a competitor, or a combination of both. As we have a unique product in our setting, we consider that only the past prices of the same brand (or product) contribute to the building of the product's reference price. This is captured by the following differential equation:

$$
\dot{R}(t)=\lambda(p(t)-R(t)) ; \quad R(0)=R_{0} \geq 0,
$$

where $R_{0}$ is the initial reference price and $\lambda \geq 0$ is a constant memory parameter capturing the reference price's speed of adjustment (i.e., indicates the strength of the impact of past retail prices on the reference price). A lower $\lambda$ indicates a smaller change in the reference price after a consumer notices a new retail-price change. According to Popescu and $\mathrm{Wu}$ (2007), this parameter may be considered as a proxy for loyalty as price memorization is affected by shopping frequency. The limit case where $\lambda=0$ corresponds to a situation where the reference price is constant.

The evolution of the reference price in equation (1) indicates that at the steady state, the retail price becomes constant and the reference price adjusts to this level. Thus, the results at the steady state under the dynamic model should be the same as those obtained under the static setting. This explains why, contrary to other papers

\footnotetext{
${ }^{6}$ Ingene et al. (2012) proved that the introduction of non-price marketing variables as control variables by channel members has a direct impact on the type of pricing scheme that can coordinate the channel.

${ }^{7}$ Retail prices may increase or decrease due to the presence or removal of promotional activities, changes in raw material prices, the introduction of new products, etc.
} 
exclusively centered on the steady-state values, we focus in this paper on the transitional dynamics and therefore characterize the optimal time paths of the main variables converging to their steady-state levels.

We follow Greenleaf (1995), Kopalle et al. (1996) and Fibich et al. (2003, 2007) by using a specification for the demand function $(Q(t))$ that captures the current and the carry-over effect of pricing decisions on demand. $Q(t)$ is given by the following expression:

$$
Q(p(t), R(t))=\alpha-\beta p(t)-\gamma(p(t)-R(t)),
$$

where $\alpha, \beta$, and $\gamma$ are positive parameters.

The reference price is introduced in the model with an additive linear effect captured by the difference between the reference price $R(t)$ and the observed retail price $p(t)$. This price discrepancy translates into a perception of a discount or a surcharge. Indeed, when $p(t)<R(t)$, consumers perceive a gain (i.e., a discount) and demand increases by the amount $|\gamma(p(t)-R(t))|$. When $p(t)>R(t)$, consumers perceive a loss (i.e., a surcharge) and the demand decreases by $|\gamma(p(t)-R(t))|$. Hence, the parameter $\gamma$ captures the magnitude of the impact on demand of this price discrepancy. A constant $\gamma$ reflects consumers' loss-neutrality, meaning that the gains or losses perceived from a lower or a higher retail price in accordance to the reference price have the same effects on demand.

Remark 1. Previous studies on reference price effects used asymmetric demand models centered on the Prospect Theory (Kahneman and Tversky, 1979). These models capture consumers' loss-aversion by considering that $\gamma$ takes higher (lower) values if $p(t)$ is higher (lower) than $R(t)$ meaning that the loss effects outweight the gain effects. ${ }^{8}$ In the present study, we restrict our interest to the scenario of loss-neutrality (i.e. the case where $\gamma$ is constant) in order to stay clear of the analytical complexities arising from the asymmetric effects of $\gamma$ and to focus on our objective of investigating the issue of channel coordination.

To answer our research questions, we have to consider two scenarios involving different channel structures: the first is a scenario of channel decentralization, where the channel members (a retailer and a manufacturer) choose their strategies $p(R)$ and $w(R)$, respectively. The second is a scenario of vertical integration, where only a retail price $p^{I}(R)$ is set by a unique decision maker (i.e., the channel owner). The optimal time paths of the reference prices resulting from both scenarios are denoted by $R(t)$ and $R^{I}(t)$, respectively.

Assuming that the unit production cost $c$ is a constant, the planning horizon is infinite, and both channel members have the same discount rate $\rho$, the objective functionals under the scenarios of vertical integration $\left(J^{I}\right)$ and decentralization $\left(J^{M}, J^{R}\right)$

\footnotetext{
${ }^{8}$ The comprehensive review of the literature on reference price by Mazumdar et al. (2005) shows that the loss aversion effect is not always supported in empirical studies.
} 
are as follows:

$$
\begin{aligned}
J^{I} & =\int_{0}^{\infty} \exp (-\rho t)(p(t)-c)[\alpha-\beta p(t)-\gamma(p(t)-R(t))] d t, \\
J^{M} & =\int_{0}^{\infty} \exp (-\rho t)(w(t)-c)[\alpha-\beta p(t)-\gamma(p(t)-R(t))] d t, \\
J^{R} & =\int_{0}^{\infty} \exp (-\rho t)(p(t)-w(t))[\alpha-\beta p(t)-\gamma(p(t)-R(t))] d t .
\end{aligned}
$$

Note that the superscripts $(I),(M)$ and $(R)$ respectively denote the profit functionals of the vertically integrated channel, the manufacturer and the retailer in the decentralized setting.

To summarize, our differential game has two players; two decision variables, $p$ and $w$; and one state variable, $R$. As the game takes place in an infinite time horizon, a standard hypothesis in the literature is to suppose that players use stationary feedbackstrategies, that is, their pricing strategies depend on the actual state of the system, i.e., the reference price.

Remark 2. Note that if we set $\gamma=0$, we remove the reference price impact on $Q(t)$. The demand function becomes linearly decreasing in $p(t)$, as previously modeled in traditional economic models. Under this scenario, the optimal retail and wholesale prices are constant and equal to those of the static formulation:

$$
\begin{aligned}
\text { Decentralization: } & w(R)=\frac{\alpha+c \beta}{2 \beta}, \quad p(R)=\frac{3 \alpha+c \beta}{4 \beta} ; \\
\text { Vertical Integration: } & p^{I}(R)=\frac{\alpha+c \beta}{2 \beta} .
\end{aligned}
$$

The value functions for the manufacturer $\left(V^{M}(R)\right)$ and the retailer $\left(V^{R}(R)\right)$ under the decentralization scenario, and for the whole channel $\left(V^{I}(R)\right)$ under vertical integration are constant and given by

$$
V^{M}(R)=\frac{(\alpha-c \beta)^{2}}{8 \beta \rho}, \quad V^{R}(R)=\frac{(\alpha-c \beta)^{2}}{16 \beta \rho}, \quad V^{I}(R)=\frac{(\alpha-c \beta)^{2}}{4 \beta \rho} .
$$

As in the static scenario, total channel profits when $\gamma=0$ are higher under vertical integration and the channel leader (i.e., the manufacturer) of the decentralized channel obtains a higher profit with respect to the follower (i.e., the retailer). Note here that the incentive for each channel member to cooperate or not with the other member depends mainly on how its individual profits compare under both scenarios. Hence, channel members that cooperate to earn the profits of a vertically integrated channel have to share the total profit $V^{I}(R)$. This can be accomplished by mechanisms that could be endogenous or exogenous (Jeuland and Shugan, 1983). We consider in this study that this mechanism is exogenous and is a result of negotiations between both channel members before the beginning of the game. We denote by $\theta \in(0,1)$ the percentage of the total profits under vertical integration that goes to the manufacturer 
and correspondingly, $1-\theta$ represents the percentage of the total profits under vertical integration that goes to the retailer. We can easily prove that the manufacturer has an incentive to coordinate his pricing strategy with the retailer only if $\theta \geq 0.5$, whereas the retailer is interested by coordination only for $\theta \leq 0.75$. For $\theta<0.5$ or $\theta>0.75$, channel coordination is not a good option for one of the channel members who then will prefer the implementation of the decentralized strategy.

\section{Results}

In this section, we compute the strategies and profits at the equilibrium under the scenarios of channel decentralization and vertical integration. Then, we compare these results at the steady state and along the optimal time-paths to provide answers

to our research questions. Finally, we illustrate some of our findings with numerical simulations and sensitivity analysis.

\subsection{Channel decentralization scenario}

Proposition 1. Under the decentralized channel structure, the retailer's reaction function is given by the following expression:

$$
p(w, R)=\frac{w}{2}+\frac{\alpha+\gamma R+\lambda\left(N_{1} R+N_{2}\right)}{2(\beta+\gamma)} .
$$

The manufacturer's and the retailer's value functions are given by

$$
\begin{aligned}
V^{M}(R) & =\frac{M_{1}}{2} R^{2}+M_{2} R+M_{3}, \\
V^{R}(R) & =\frac{N_{1}}{2} R^{2}+N_{2} R+N_{3},
\end{aligned}
$$

where $M_{1}, M_{2}, M_{3}, N_{1}, N_{2}$ and $N_{3}$ are positive constants given in Appendix A.

Proof. See Appendix A.

The first result in Proposition 1 indicates that the retail price is positively affected by the transfer price, indicating that the decisions of both channel members are strategic complements. Thus, if one of the channel members increases (reduces) the price at his level, the other channel member will react by increasing (decreasing) his price as well.

The second result states that the channel members' value functions are linearquadratic, which is an expected result for this type of linear-quadratic games. Note that the retailer's and the manufacturer's value-function parameters are linked in the following manner:

$$
N_{1}=\frac{M_{1}}{2}, \quad N_{2}=\frac{M_{2}}{2}, \quad N_{3}=\frac{M_{3}}{2} .
$$

Therefore, we have that

$$
V^{R}(R)=\frac{1}{2} V^{M}(R)
$$


This result coincides with previous results obtained for bilateral monopolies in a static setting: the channel leader obtains twice the profits of the follower in a decentralized channel (Ingene et al. 2012, p.19).

Assumption 1. From now on, the following condition is assumed to be satisfied:

$$
Q(c, 0)=\alpha-c(\beta+\gamma)>0
$$

This assumption means that the demand is positive for the minimum retail price (equal to the production cost, $c)$ and the minimum reference price $(R=0)$.

Coefficient $M_{1}$ is always positive and under Assumption 1, it can be proven that $M_{2}$ is also positive and that $M_{3}$ is thus positive.

Proposition 2. Transfer and retail prices at the equilibrium under the decentralized channel structure are given by

$$
\begin{aligned}
& w(R)=\frac{2(\alpha+c(\beta+\gamma))+\lambda M_{2}+\left(2 \gamma+\lambda M_{1}\right) R}{4(\beta+\gamma)} \\
& p(R)=\frac{2(3 \alpha+c(\beta+\gamma))+3 \lambda M_{2}+3\left(2 \gamma+\lambda M_{1}\right) R}{8(\beta+\gamma)} .
\end{aligned}
$$

Proof. See Appendix A.

The above proposition states that the transfer price and the retail price under the decentralized channel are linear and increasing in the state variable, indicating that when the consumers' reference price increases, both the manufacturer and the retailer increase their transfer and retail prices, to benefit from an increase in the consumers' willingness to pay.

Furthermore, the result indicates that any increase in the reference price has a stronger effect on the retail price rather than on the transfer price because $w^{\prime}(R)<$ $p^{\prime}(R)$ for any value of $R$. This result is mainly because any variation in the reference price has an effect on the transfer price as well as on the retail price. However, as the transfer price contributes to the increase in the retail price, the latter is subject to twice the effect of the reference-price variation (i.e., a direct effect due to the link between $p$ and $R$ and an indirect effect due to the effect of $R$ on $w$ and the effect of $w$ on $p$ ).

Proposition 3. Under the decentralized scenario, the reference price at the steady state is given by

$$
R_{s s}=\frac{6 \alpha+2 c(\beta+\gamma)+3 M_{2} \lambda}{8 \beta+2 \gamma-3 M_{1} \lambda}=p_{s s} .
$$

The steady state is positive and globally stable.

Furthermore, under Assumption 1, $R_{s s}$ increases with $\alpha$ and $c$, but decreases with $\beta$ and $\gamma$. 
Proof. See Appendix A.

The results of the above proposition indicate that an increase in parameter $\alpha$ leads to an increase in the reference price at the steady state. Note that parameter $\alpha$ captures the baseline sales that can be interpreted as a measure of brand equity. Thus, this result indicates that an increase in brand equity results in an increase in the willingness to pay for a brand.

Regarding the effect of parameter $c$, we expect a positive relationship between unitproduction cost and retail price, meaning that higher production costs lead to higher retail prices and consequently to higher reference prices at the steady state.

The negative impact of $\beta$ on the reference price at the steady state also has an intuitive interpretation because we expect that a stronger negative effect by retail prices on demand will force the retailer to reduce his retail price thereby negatively affecting the reference price in the long run.

Furthermore, the negative impact of $\gamma$ on the level of the reference price at the steady state indicates that the reference price in the long run decreases when its effect on demand increases. This result is also intuitive and confirms the previous result found in Benchekroun et al. (2009) and Popescu and Wu (2007). Indeed, we expect that an increase in the reference-price effect on demand will give the retailer an incentive to decrease his retail price to reach a level lower than that of the reference price. This leads to a decrease in the reference price as well because of the system dynamics.

\subsection{Vertical integration scenario}

Proposition 4. The optimal retail price for the vertically integrated channel is given by the expression

$$
p^{I}(R)=\frac{\alpha+c(\beta+\gamma)+\gamma R+\lambda\left(I_{1} R+I_{2}\right)}{2(\beta+\gamma)},
$$

and the value function of the vertically integrated channel is given by

$$
V^{I}(R)=\frac{I_{1}}{2} R^{2}+I_{2} R+I_{3},
$$

where $I_{1}, I_{2}$ and $I_{3}$ are positive constants given in Appendix B.

Proof. See Appendix B.

Expression $I_{1}$ is positive and under Assumption $1, I_{2}$ can also easily be proved positive; thus, $I_{3}$ is positive as well.

The results under this proposition are qualitatively similar to the results obtained under the scenario of channel decentralization. We obtain that the retail price is linear in the state variable, that an increase in the reference price leads to an increase in the retail price, and that the total channel value function is linear-quadratic in the state variable.

Proposition 5. Under vertical integration, the reference price at the steady state is given by

$$
R_{s s}^{I}=\frac{\alpha+c(\beta+\gamma)+I_{2} \lambda}{2 \beta+\gamma-I_{1} \lambda}=c+\frac{(\alpha-c \beta)(\lambda+\rho)}{\gamma \rho+2 \beta(\lambda+\rho)} .
$$


The steady state is positive and globally stable.

Furthermore, under Assumption 1, $R_{\text {ss }}^{I}$ increases with $c, \alpha$ and $\lambda$, but decreases with $\beta, \rho$ and $\gamma$.

Proof. See Appendix B.

The same interpretations as for the decentralized case apply to the effect of parameters $c, \alpha, \beta$ and $\gamma$. Here we have two additional parameters $(\lambda$ and $\rho$ ) for which we obtain a result on their impact on the reference price at the steady state.

Our findings suggest that the retail price at the steady state increases when the memory parameter $\lambda$ increases. This result confirms previous findings in Popescu and $\mathrm{Wu}(2007)$ for the loss-neutral buyer scenario, when they examine the impact of this parameter on retail prices, which adjusts to the reference price at the steady state. According to the authors, if, under this scenario, we consider consumers to be heterogeneous with respect to the memory parameter, then firms should charge lower steady-state retail prices for consumers presenting a low memory parameter (all else being equal). Although this pricing policy is not consistent with current practices in the industry, where firms tend to reward loyal customers by offering them more tempting price reductions, it appears to be the optimal strategy for some situations.

Furthermore, our results regarding the negative impact of the discount rate $(\rho)$ on the steady-state reference price are similar to the findings of Nasiry and Popescu (2011). These results state that retail prices at the steady state are higher for low values of the discount rate $(\rho)$, suggesting that, contrary to patient firms (small $\rho$ ) that charge high steady-state retail prices, a myopic firm (high $\rho$ ) ends up with a lower steady-state retail price and thus a lower reference price at the steady state.

This section was devoted to the computation of channel members' strategies at the equilibrium under the two channel structure scenarios. The above propositions expose the different analytical results obtained with our dynamic model. Although some of the results under both scenarios could be compared analytically, most of the results are characterized by huge and complicated expressions and the most appropriate approach in that case is numerical illustration.

\subsection{Comparing scenarios}

In this section, we compare our findings under the scenarios of channel decentralization and vertical integration. We start by comparing results at the steady state, and then we examine the results for the transient pricing policy before reaching the steady state.

\subsubsection{At the steady state}

As mentioned previously, the results obtained with our model at the steady state coincide with those obtained under a static setting. This result is expected as the specification of our model states that at the steady state, the reference price adjusts to the retail price, and thus, the effect of the differential term $(p(t)-R(t))$ vanishes in both equations (1) and (2). Hence, our model becomes similar to previous models identified 
in the static literature on pricing in marketing channels (see Jeulang and Shugan (1983) and Ingene et al. (2012)) and confirms the efficiency of vertical integration. ${ }^{9}$

Indeed, we can prove analytically that

$$
\operatorname{sign}\left(R_{s s}^{I}-R_{s s}\right)=-\operatorname{sign}(\alpha-c \beta) .
$$

Under Assumption 1, $\alpha-c \beta$ is positive, and therefore,

$$
R_{s s}^{I}<R_{s s} .
$$

Because at the steady state, the retail and reference prices coincide,

$$
p_{s s}^{I}<p_{s s} .
$$

At the steady state, the demands under both scenarios read:

$$
Q_{s s}^{I}=\alpha-\beta p_{s s}^{I}, \quad Q_{s s}=\alpha-\beta p_{s s} .
$$

Thus,

$$
Q_{s s}^{I}>Q_{s s} .
$$

By virtue of joint optimization: $V_{R s s}^{I}+V_{M s s}^{I} \geq V_{R s s}+V_{M s s}$.

As mentioned above, all of these results are, as expected, similar to those obtained under the static setting. However, it is not clear whether they can be always reproduced along the whole optimal time-path, that is, all along the transient pricing policy. Observing channel members strategies and profits along the optimal time-paths before reaching the steady state is particularly important in the context of marketing channels as channel members do not have always binding agreements nor behave systematically in a farsighted manner. As a consequence, a channel member that computes its cumulative individual profit in an intermediary period during the transient trajectory to the steady state could be tempted to end its cooperation with its partner if he finds out that profits under decentralization are higher than its share of total profits in the vertically integrated scenario. In a static setting and at the steady state, channel members do not have the option of deviation that is associated with the transient period when the reference price is adjusting to its steady-state value and that underlines the dynamic aspect of the problem.

In the next section, we compute the time-paths of channel members strategies and profits to compare the results under both scenarios.

\footnotetext{
${ }^{9}$ It is worth noting that here again, both channel members are not necessarily interested in the implementation of the vertically integrated solution at the steady state although the total channel profits under this scenario are higher with respect to decentralization. As in the static case, this choice will depend on the values of their total channel profit shares, $\theta$ and $(1-\theta)$, respectively.
} 


\subsubsection{Results for the optimal time-paths}

The following proposition shows that if the initial reference price is lower than the steady-state value $R_{s s}^{I}$, there exists some time interval along which the optimal timepath of the reference price under decentralization is lower than its corresponding value under vertical integration.

Proposition 6. There is an initial time period in which the reference price under the channel decentralization scenario is lower than the reference price under vertical integration. This phenomenon is observed only for values of the initial reference price that are lower than $R_{s s}^{I}$.

Proof. The expressions of $R(t)$ and $R^{I}(t)$ are obtained from the integration of differential equation (1) once the optimal strategy of the retail price has been replaced for both scenarios, and are given by

$$
\begin{aligned}
R(t) & =\left(R_{0}-R_{s s}\right) e^{-B t}+R_{s s} \\
R^{I}(t) & =\left(R_{0}-R_{s s}^{I}\right) e^{-B^{I} t}+R_{s s}^{I}
\end{aligned}
$$

where

$$
B=\frac{\lambda\left(8 \beta+2 \gamma-3 \lambda M_{1}\right)}{8(\beta+\gamma)}>0, \quad B^{I}=\frac{\lambda\left(2 \beta+\gamma-\lambda I_{1}\right)}{2(\beta+\gamma)}>0 .
$$

It can be proved that $\frac{\gamma}{2}+\lambda\left(\frac{3}{4} M_{1}-I_{1}\right)>0$ and then, $B<B^{I}$. Consequently, the reference price time-paths for both scenarios compare as follows:

- If $R_{0}>R_{s s}^{I}$, then $R(t)>R^{I}(t)$ for all $t>0$.

- If $R_{0}<R_{s s}^{I}$, then there exists $\tilde{t}>0$ such that $R(t)<R^{I}(t)$ for $t \in(0, \tilde{t})$ and $R(t) \geq R^{I}(t)$ for $t \geq \tilde{t}$.

The above proposition gives a surprising but interesting result from a marketing channel perspective. Indeed, for some low initial reference-price values, there is an initial time period where consumers' price expectations are higher under vertical integration than under decentralization. This result is surprising as decentralization in bilateral monopolies should lead to double marginalization. ${ }^{10}$ Thus, consumers should expect to pay a higher price when they purchase products from an intermediary instead of buying directly from the producer. This result is explained by the results obtained in the next proposition.

Proposition 7. Depending on the initial value of the reference price, and consequently, on the initial value of the retail price, there are time periods during which the retail price under the vertically integrated channel is higher than the retail price under decentralization. This result can be observed not only when the initial value of the retail

\footnotetext{
${ }^{10}$ This result has been proved in previous studies. See, for example, Jeuland and Shugan (1983).
} 
price under decentralization is lower than the initial value under vertical integration (case 4 below) but also in other cases, even if the initial values of the retail price under the two scenarios compare the other way around (cases 2.6 and 3.6 below).

Proof. The optimal retail-price equilibrium strategies under decentralization and vertical integration are given by equations (11) and (13), respectively. Let us denote by $p_{0}$ and $p_{0}^{I}$ the initial values of the retail prices for both scenarios. Their expressions are given by replacing $R$ by its initial value $R_{0}$ :

$$
\begin{aligned}
& p_{0}=C R_{0}+\frac{6 \alpha+2 c(\beta+\gamma)+3 M_{2} \lambda}{8(\beta+\gamma)}, \\
& p_{0}^{I}=C^{I} R_{0}+\frac{\alpha+c(\beta+\gamma)+I_{2} \lambda}{2(\beta+\gamma)},
\end{aligned}
$$

where

$$
C=\frac{3\left(2 \gamma+M_{1} \lambda\right)}{8(\beta+\gamma)}>0, \quad C^{I}=\frac{\gamma+I_{1} \lambda}{2(\beta+\gamma)}>0 .
$$

The comparison between the optimal retail price time-paths under the decentralized and the vertically integrated scenarios depends on the initial values $p_{0}$ and $p_{0}^{I}$, given in (19) and (20), in terms of the initial value of the reference price, $R_{0}$. Indeed:

1. If $p_{0}^{I}<p_{s s}^{I}<p_{0}$ or $p_{s s}^{I}<p_{0}^{I}<\min \left\{p_{0}, p_{s s}\right\}$, then $p(t)>p^{I}(t)$ for all $t \geq 0$.

2. If $p_{0}^{I}<p_{0}<p_{s s}^{I}$, then two possibilities can arise:

(a) if $\dot{p}^{I}(0)<\dot{p}(0)$, then $p(t)>p^{I}(t)$ for all $t \geq 0$.

(b) if $\dot{p}^{I}(0)>\dot{p}(0)$, then there exists $K>0$ such that if $p_{0}^{I}<p_{0}<\min \left\{p_{0}^{I}+\right.$ $\left.K, p_{s s}^{I}\right\}$, there exist $\tilde{t}, \tilde{\tilde{t}}>0$ such that $p(t) \geq p^{I}(t)$ for $t \in[0, \tilde{t}], p(t)<p^{I}(t)$ for $t \in(\tilde{t}, \tilde{\tilde{t}})$, and $p(t) \geq p^{I}(t)$ for $t \geq \tilde{\tilde{t}}$.

3. If $p_{s s}<p_{0}^{I}<p_{0}$, then two possibilities can arise:

(a) if $0>\dot{p}(0)>\dot{p}^{I}(0)$, then $p(t)>p^{I}(t)$ for all $t \geq 0$.

(b) if $0>\dot{p}^{I}(0)>\dot{p}(0)$, then there exists $L>0$ such that if $\max \left\{p_{0}-L, p_{s s}\right\}<$ $p_{0}^{I}<p_{0}$, there exist $\hat{t}, \hat{\hat{t}}>0$ such that $p(t) \geq p^{I}(t)$ for $t \in[0, \hat{t}], p(t)<p^{I}(t)$ for $t \in(\hat{t}, \hat{\hat{t}})$, and $p(t) \geq p^{I}(t)$ for $t \geq \hat{\hat{t}}$.

4. If $p_{0}<p_{0}^{I}$, then there exists $\bar{t}>0$ such that $p(t)<p^{I}(t)$ for $t \in[0, \bar{t})$, and $p(t) \geq p^{I}(t)$ for $t \geq \bar{t}$.

Remark 3. Let us note that $\dot{p}^{I}(0)$ can be greater or less than $\dot{p}(0)$.

The following equivalence can be easily deduced:

$$
\dot{p}^{I}(0)>\dot{p}(0) \Leftrightarrow C^{I}\left(R_{0}-R_{s s}^{I}\right) B^{I}<C\left(R_{0}-R_{s s}\right) B .
$$

Recall that $B^{I}>B$, and $R_{s s}^{I}<R_{s s}$, and then, $R_{0}-R_{s s}^{I}>R_{0}-R_{s s}$. It can be proved that $C>C^{I}$, and therefore, $\dot{p}^{I}(0)$ can be greater or less than $\dot{p}(0)$. 
The results in the above proposition indicate that there are some situations (cases 2.b, 3.b and 4) where the retail price of a vertically integrated channel could be higher than the retail price of a decentralized channel. This result is the first to prove that with the introduction of dynamic features into the pricing model, one can find situations where double-marginalization does not lead to higher prices. This result applies for finite periods of time that, in cases $2 . \mathrm{b}$ and $3 . \mathrm{b}$, correspond to intermediate periods of time, whereas in case 4 , it applies only to an initial time interval.

Corollary 2. If the initial value of the reference price is lower than an upper bound denoted by $\tilde{R}_{0}$, then there is an initial time period along which both the retail and reference prices under decentralization are lower than their counterparts under vertical integration.

\section{Proof.}

Let us first completely characterize the comparison of the initial retail prices under both scenarios, decentralization and vertical integration, $p_{0}, p_{0}^{I}$, respectively. Notice that

$$
p_{0}<p_{0}^{I} \Leftrightarrow C R_{0}+\frac{A}{\lambda}<C^{I} R_{0}+\frac{A^{I}}{\lambda} \Leftrightarrow\left(C-C^{I}\right) R_{0}+\frac{A-A^{I}}{\lambda}<0,
$$

where

$$
A=\lambda \frac{6 \alpha+2 c(\beta+\gamma)+3 M_{2} \lambda}{8(\beta+\gamma)}, \quad A^{I}=\lambda \frac{\alpha+c(\beta+\gamma)+I_{2} \lambda}{2(\beta+\gamma)} .
$$

From the previous equivalence, and recalling that $C-C^{I}>0$, depending on the values of $A$ and $A^{I}$, the initial values of the retail prices for both scenarios compare as follows:

- If $A>A^{I}$, then $p_{0}>p_{0}^{I}$.

- If $A<A^{I}$ and $\tilde{R}_{0}=-\frac{A-A^{I}}{\lambda\left(C-C^{I}\right)}$, we have

* if $R_{0}>\tilde{R}_{0}$, then $p_{0}>p_{0}^{I}$;

$*$ if $R_{0}<\tilde{R}_{0}$, then $p_{0}<p_{0}^{I}$.

Let us note that the steady-state values of the reference price can be rewritten as

$$
R_{s s}=\frac{A}{B}, \quad R_{s s}^{I}=\frac{A^{I}}{B^{I}}
$$

It can be easily proved that

$$
\tilde{R}_{0}<R_{s s}^{I} .
$$

Therefore, if condition $R_{0}<\tilde{R}_{0}$ is satisfied, on the one hand, $p_{0}<p_{0}^{I}$ (because $A<A^{I}$ ), and as Case 4 in Proposition 7 states, there is an initial interval of time $[0, \bar{t})$ such that $p(t)<p^{I}(t)$ for $t \in[0, \bar{t})$. On the other hand, condition $R_{0}<\tilde{R}_{0}$ from $(21)$ establishes $R_{0}<R_{s s}^{I}$, implying that there is an initial interval of time $(0, \tilde{t})$ such that $R(t)<R^{I}(t)$ for $t \in(0, \tilde{t})$ (see the last line in the proof of Proposition 6). 
The above results stress the importance of taking into account not only the dynamics regarding the evolution of retail prices and their impact on the reference price and thus the demand but also the importance of considering the level of the initial reference price, $R_{0}$. Indeed, this value leads to different levels of the initial retail price fixed by firms whether they are vertically integrated or decentralized and thus affects the evolution of consumers' price expectations and the pricing strategies that these firms can adopt during their planning horizon.

\subsection{Numerical illustrations}

To illustrate our analytical results and to highlight the importance of adopting a dynamic perspective in the study of pricing decisions in channels, we run some numerical simulations.

We fixed the following values for the parameters of the benchmark case:

$$
c=0, \alpha=1, \beta=1 \text {, and } \rho=0.1 \text {. }
$$

We kept parameters $\gamma$ and $\lambda$ variable, as they capture the impact of the differential term $(p(t)-R(t))$, which is responsible for the dynamic features of the model.

We focused on the characterization of the parameter values for which $p_{0}<p_{0}^{I}$ because under this condition, there is always an initial interval of time $([0, \bar{t}))$ in which $p(t)<p^{I}(t)$. Therefore, as previously stated, we need to ensure that $A<A^{I}$, and for $R_{0}<\tilde{R}_{0}=-\frac{A-A^{I}}{\lambda\left(C-C^{I}\right)}$, then inequality $p_{0}<p_{0}^{I}$ applies.

Expression $A-A^{I}$ is independent of the value of $\alpha$, and its sign depends on the values of $\gamma$ and $\lambda$ as shown below.

- For $\gamma \in[0,2], A>A^{I}$ for any value of $\lambda$.

- For $\gamma \geq 3$, then $A<A^{I}$ if and only if ${ }^{11} \lambda>\tilde{\lambda}$.

These results indicate that the value of $\gamma$ has an impact on the time horizon during which the decentralized retail price could be lower than the vertically integrated price. Indeed, we can observe that for low values of $\gamma$, the decentralized retail price can never be lower than the vertically integrated at the beginning of the planning horizon, whereas the situation could be observed later on during the planning horizon. When the value of $\gamma$ is increased, we notice that, under a constraint on the minimum level of the parameter $\lambda$, a decentralized retail price is lower than the vertically integrated one only at the beginning of the planning horizon.

The value of $\tilde{R}_{0}$ is proportional to $\alpha$, then $\alpha$ can be viewed as a scale parameter. We have fixed $\alpha=1$, but the value of $\tilde{R}_{0}$ could be increased (decreased) by fixing $\alpha>1$ $(\alpha<1)$. The value of $\tilde{R}_{0}$ increases as $\lambda$ or $\gamma$ increases. Let us recall that both $R_{s s}^{I}$ and $R_{s s}$ increase as $\lambda$ increases but decrease as $\gamma$ increases.

The numerical simulations also reveal that the larger the value of $\lambda$ or $R_{0}$ is, the shorter the initial time period $[0, \bar{t})$ such that $p(t)<p^{I}(t)$ for $t \in[0, \bar{t})$. These results

\footnotetext{
${ }^{11}$ The value of $\tilde{\lambda}$ decreases as $\gamma$ increases.
} 
indicate that, if consumers' loyalty is low (low $\lambda$ ), the time horizon during which the decentralized channel fixes its retail price at a lower level (with respect to its level under a vertically integrated channel) is longer. The same result is observed for low values of the initial reference price.

Furthermore, we ran additional numerical simulations ${ }^{12}$ to investigate if our results still hold if we change the parameters $\alpha, \beta, \rho$ and $\gamma$. Our findings indicate that a lower discount rate pushes channel members into setting lower retail prices under decentralization (compared to their counterparts under vertical integration) during a longer time period. When the discount rate is high, the retail price under decentralization increases faster to exceed the retail price under vertical integration. The time horizon during which the decentralized price is lower than the coordinated retail price becomes shorter for high values of the parameter $\beta$ (i.e., the short-term impact of the retail price on demand). Our simulations indicate that this time period becomes longer with an increase in the parameter $\gamma$. We note that if we focus only on the immediate effects of retail prices on demand (by fixing a high $\beta$ and a low $\gamma$ ), we will mainly observe situations where the cooperative retail price is lower than the decentralized retail price, as already proven in the static literature. In the opposite situation where we increase the long-term impact of the retail price (by fixing a higher $\gamma$ ), we can find time intervals where the decentralized retail price is lower than the coordinated one.

We also note that the initial retail-price values under decentralization and vertical integration, $p_{0}, p_{0}^{I}$ increase with $R_{0}$, as well as with $\lambda$.

Let us consider the following example, $\gamma=10$ and $\lambda=100$. The steady-state values of the reference price for both scenarios $\left(R_{s s}, R_{s s}^{I}\right)$ and the bound $\tilde{R}_{0}$ are given by

$$
R_{s s}=0.583679, \quad R_{s s}^{I}=0.497515, \quad \tilde{R}_{0}=0.308998 .
$$

Let us fix the initial value of reference price $R_{0}=0.3$. With these data, the initial retail-price values in both scenarios are

$$
p_{0}=0.358781, \quad p_{0}^{I}=0.359633 .
$$

Finally, the value of $\bar{t}$ can be computed numerically:

$$
\bar{t}=0.00163796 \text {. }
$$

\subsubsection{Skimming and Penetration strategies in distribution channels}

One of the questions addressed in this paper is whether the adoption of a skimming or a penetration strategy is affected by the distribution channel's structure (i.e., a decentralized versus a vertically integrated channel). In our model, we capture the dynamic effects of pricing strategies by considering that retail prices contribute to the building of a reference price used by consumers as a benchmark to make their purchase decisions. Hence, when fixing their retail prices, firms should take into account

\footnotetext{
${ }^{12}$ The results for this sensitivity analysis are not displayed in the paper. The corresponding author can provide these results for the interested readers.
} 
consumers' reference prices and examine how these prices compare. If the consumers' initial reference price is low, then the firm must fix a low retail price at the introductory stage and then increase it until the price reaches the steady-state value (penetration strategy). In the opposite situation, if the initial reference price is high, the firm should adopt a skimming strategy where retail prices are fixed at high levels and then decreased later on to their steady-state levels (Fibich et al. 2003; Popescu and Wu, 2007).

To answer our third research question, we performed some numerical simulations where we considered different initial values for the reference price and the values of the parameters were fixed to: $c=0, \alpha=1, \beta=1, \rho=0.1, \gamma=10, \lambda=100$. We fixed three different levels for the initial reference price $R_{0} \in\{0.3,0.55,0.8\}$. With these values, we were able to observe three different scenarios, depending on the position ${ }^{13}$ of $R_{0}$ with respect to $R_{s s}^{I}$ and $R_{s s}$. For each scenario, we plotted the retail prices under the decentralized channel and the vertically integrated channel, and the values of the reference prices at the steady state for both channel structures (these values coincide with the retail prices at the steady state).

Our results can be summarized as follows:

- For all of the scenarios we investigated, the firm's transient pricing policy always indicates a monotone pricing policy, meaning that firms either use a skimming or a penetration strategy but never use both, in their transition to the steady-state level. In all situations, the pricing policies always converge to the steady-state level of the reference price, regardless of the reference price's initial level. This behavior was also observed by Kopalle et al. (1996) and Fibich et al. (2003).
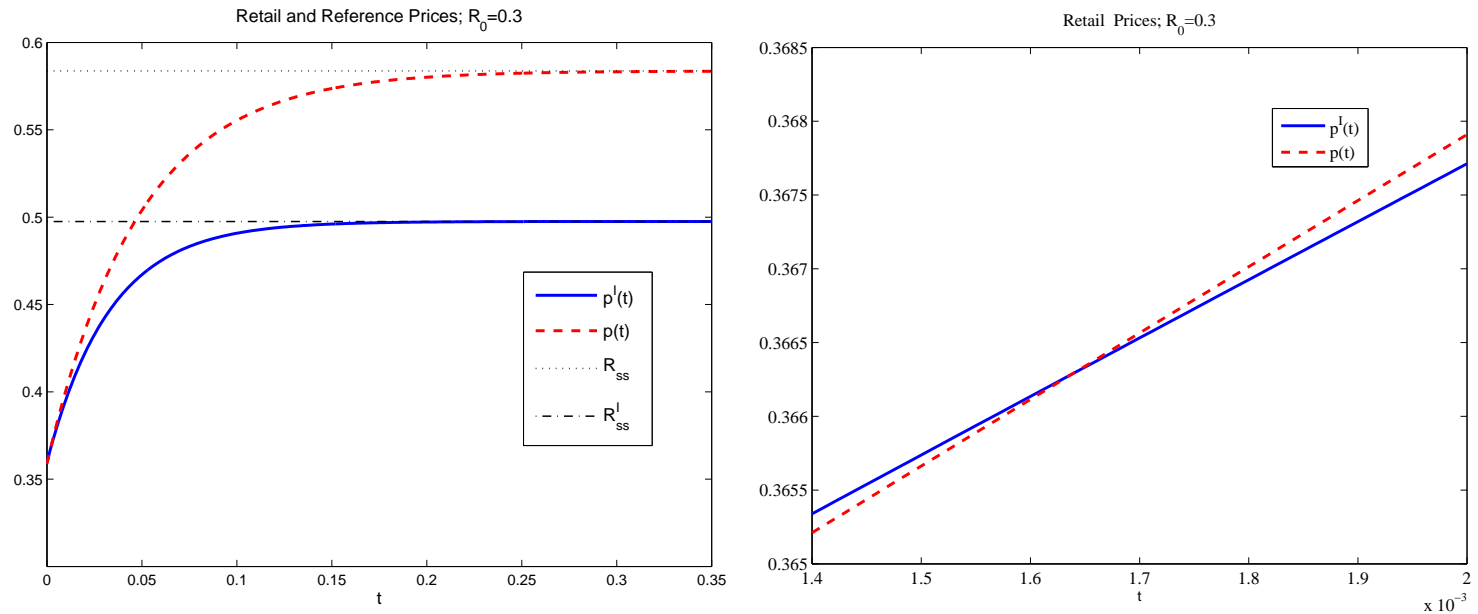

Figure 1: Comparison of retail prices: $R_{0}=0.3$ (left); Zoom for $t \in[0.0014,0.002]$ (right)

- For the scenario where $R_{0} \leqslant R_{s s}^{I} \leqslant R_{s s}$ (we denote it by the low initial referenceprice scenario) we found that this is the only scenario where the instantaneous

\footnotetext{
${ }^{13}$ Recall that we always have that $R_{s s}^{I} \leq R_{s s}$.
} 
retail price could be higher under vertical integration than under channel decentralization (see Figures 1 left and right for $R_{0}=0.3$ ). This occurs only for the initial period of time computed previously $(t \in[0, \bar{t}=0.00163796))$ (see the zoom for $t \in[0.0014,0.002]$ in Figure 1 right). For this same period, we also found that the instantaneous profit under vertical integration is lower than the profit under decentralization. Our numerical simulations indicate that this initial interval becomes shorter for higher values of the memory parameter $(\lambda)$ and for higher values of $R_{0}$. This last result is expected because higher values of $R_{0}$ allow us to move to the other scenarios (medium and high values of the initial reference price), where the result of the comparison of $p(t)$ and $p^{I}(t)$ is reversed.

For this scenario, regardless of the channel's structure, the best dynamic pricing strategy is penetration. Such a situation results in the perception of a price surcharge (loss) by consumers during the introductory stage, as the retail price before reaching the steady state is higher than the reference price. This result is in line with previous findings by Popescu and $\mathrm{Wu}$ (2007).

- For the scenario where $R_{s s}^{I} \leq R_{0} \leq R_{s s}$ (denoted by the medium initial referenceprice scenario) our results indicate that the instantaneous retail price under decentralization is higher than its counterpart in the vertically integrated scenario $\left(p(t) \geqslant p^{I}(t)\right)$. This result contrasts the result obtained under the previous scenario, but coincides with previous findings in the static literature. Furthermore, we find that a decentralized channel practices a penetration strategy whereas a vertically integrated channel uses price skimming (see Figure 2 left for $R_{0}=0.55$ ). Thus, consumers perceive a deal under vertical integration and a price surcharge under channel decentralization.
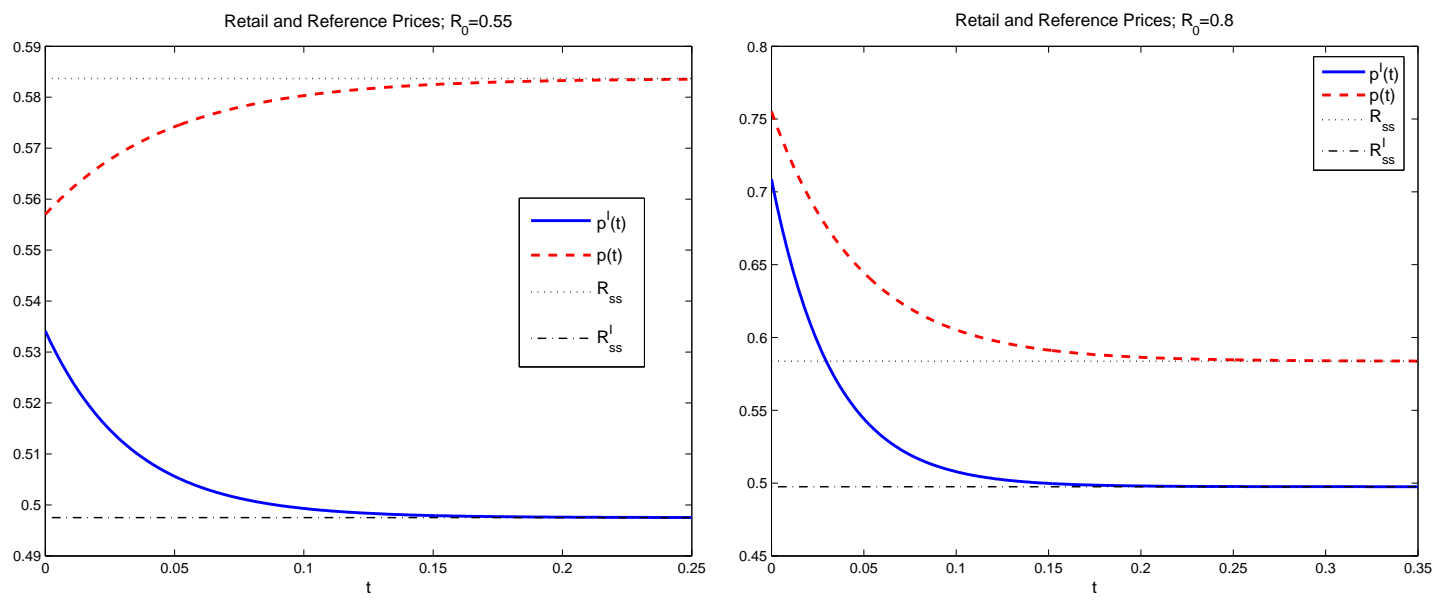

Figure 2: Comparison of retail prices: $R_{0}=0.55$ (left); $R_{0}=0.8$ (right)

- For the scenario where $R_{s s}^{I} \leq R_{s s} \leq R_{0}$ (denoted by the high initial referenceprice scenario) we still have $p(t) \geqslant p^{I}(t)$ as in the medium initial reference-price scenario. However, our results indicate that price skimming is practiced regardless 
of the type of channel structure (see Figure 2 right for $R_{0}=0.8$ ). This scenario leads to the opposite results with respect to those obtained under the scenario of a low initial reference price. They also confirm previous findings by Popescu and $\mathrm{Wu}$ (2001). Indeed, under this situation, consumers always perceive a deal, regardless of whether the channel is vertically integrated or decentralized.

Remark 4. The instantaneous consumer surplus along the optimal paths of the retail and reference prices under vertical integration $\left(C S^{I}(t)\right)$ and under decentralization $(C S(t))$ are given by

$$
\begin{aligned}
C S^{I}(t) & =\left[\frac{1}{2(\beta+\gamma)}\left(\alpha+\gamma R^{I}(t)\right)-p^{I}(t)\right]\left(\alpha+\gamma R^{I}(t)\right)+\frac{\beta+\gamma}{2}\left(p^{I}\right)^{2}(t), \\
C S(t) & =\left[\frac{1}{2(\beta+\gamma)}(\alpha+\gamma R(t))-p(t)\right](\alpha+\gamma R(t))+\frac{\beta+\gamma}{2}(p)^{2}(t) .
\end{aligned}
$$

For the low initial reference-price scenario $\left(R_{0}=0.3\right)$, the instantaneous consumer surplus is greater under decentralization than under vertical integration for an initial interval of time $(t \in[0,0.0016))$, whereas the opposite applies for any time greater than $^{14} t=0.0016$.

However, for the medium and high initial reference-price scenarios $\left(R_{0}=0.55\right.$ and $R_{0}=0.8$, respectively) the instantaneous consumer surplus is greater under vertical integration than under decentralization along the whole time horizon.

As for the static case, our results at the steady state indicate that consumer surplus is higher under vertical integration.

\subsubsection{Channel profits along the optimal time-paths}

In this section, for the same parameter values as indicated before, we computed and compared time-paths of the total channel profits under the different scenarios (denoted by $T P(t)$ and $T P^{I}(t)$ for decentralization and vertical integration, respectively) corresponding to the different values of the initial reference-price levels $\left(R_{0}\right)$. We also discuss the implications of examining the issue of channel coordination from a dynamic perspective to highlight the importance of our findings from a managerial perspective.

Figures 3 and 4 indicate the following results:

- For low values of $R_{0}$, the time paths of the total channel profits increase during the planning horizon, and, along the optimal trajectory, vertical integration performs better than decentralization (see Figure 3 left).

- For medium levels of $R_{0}$, the time paths of the total channel profits evolve in opposite directions (see Figure 3 right). Indeed, the time paths of the total channel profits decreases under vertical integration but increase under decentralization. Vertical integration still performs better than decentralization during the whole planning horizon, but the discrepancy between both profits decreases with time.

\footnotetext{
${ }^{14}$ Note that this value corresponds to the planning horizon under which the retail price under decentralization is lower than the retail price under vertical integration.
} 

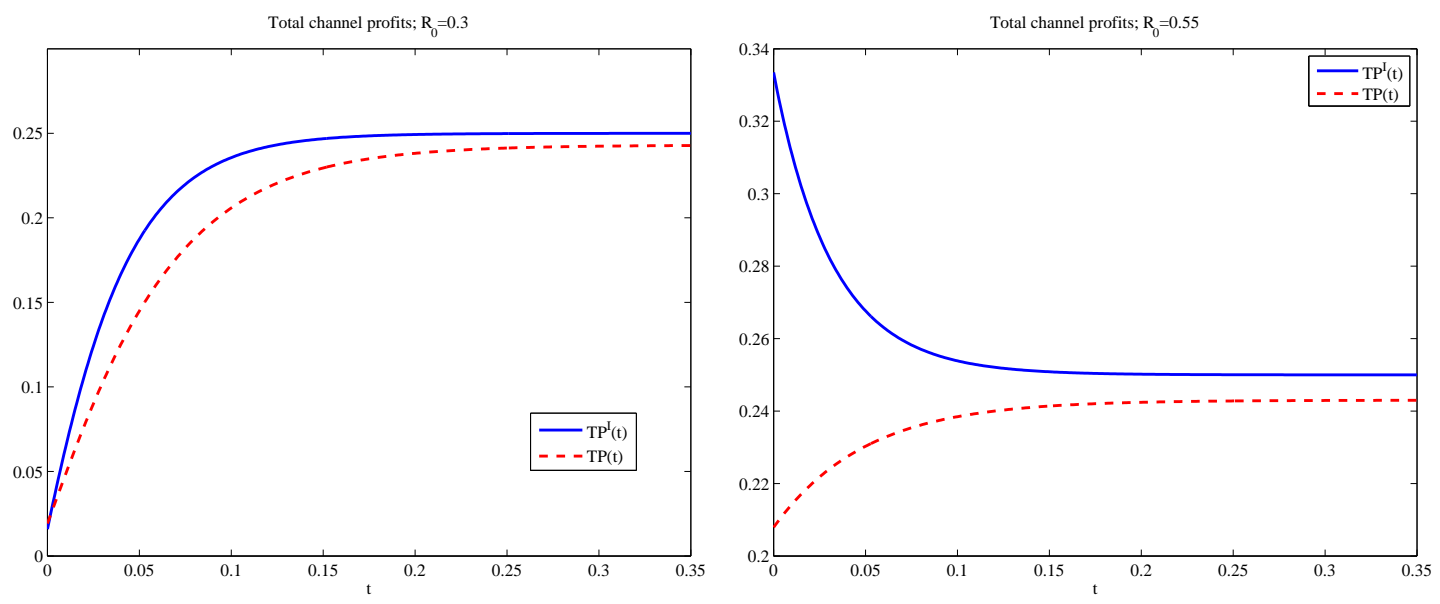

Figure 3: Comparison of total channel profits: $R_{0}=0.3$ (left); $R_{0}=0.55$ (right)

- For high values of $R_{0}$, our results indicate a decrease in the time paths of the total channel profits under both scenarios of vertical integration and decentralization (see Figure 4 left). We note that for a short time period (starting at $\tilde{t}=0.0939$ ), decentralization performs better than vertical integration (see Figure 4 right). This result is of interest because it indicates that under a dynamic setting, vertical integration may be inefficient and that channel members involved in a vertically integrated structure could be tempted to act non-cooperatively during this short time period if they have myopic behavior.
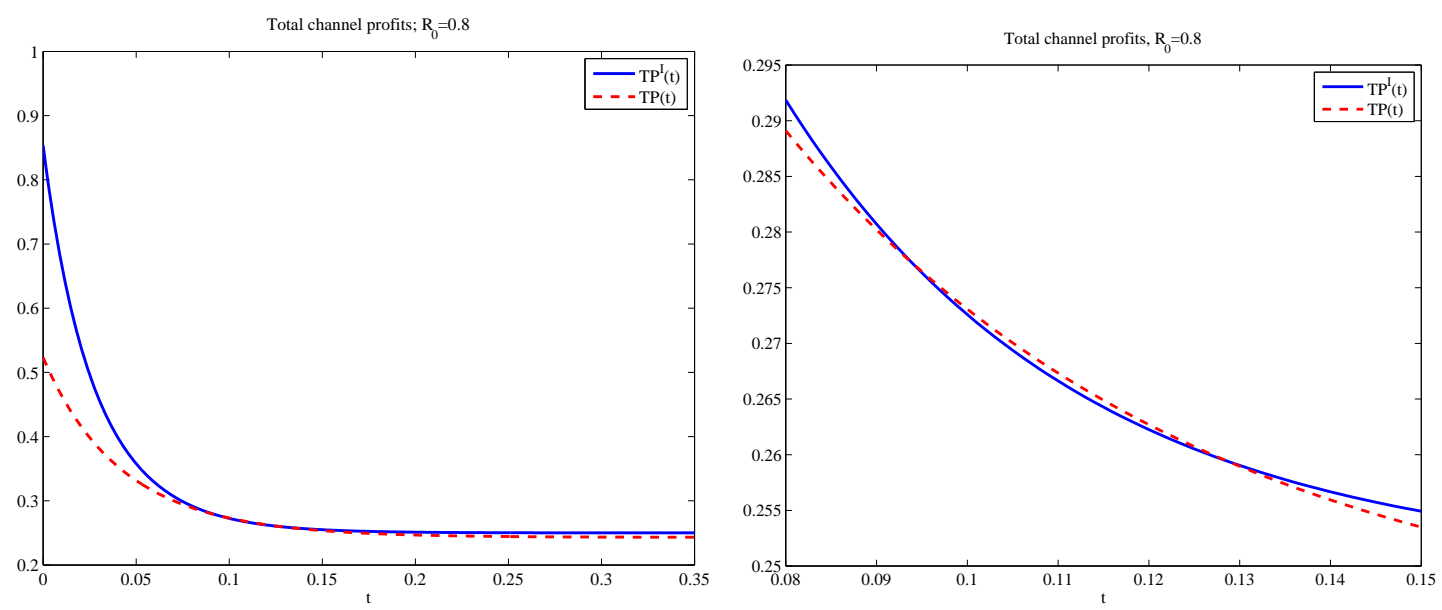

Figure 4: Comparison of total channel profits: $R_{0}=0.8$ (left); Zoom for $t \in[0.08,0.015]$ (right)

Although the time interval during which a decentralized channel performs better than a coordinated channel is a short period (with respect to the whole planning horizon), the channel members still have the temptation to behave in a uncoordinated manner, especially if there is no binding agreement that forces them to pursue their cooperation. 
Indeed, by examining the pricing issue in distribution channels from a dynamic perspective, we allow channel members to evaluate their strategies and profits at any point in time during the planning horizon. Depending on their valuation of actual versus future profits, channel members could behave differently:

- Each channel member could, at the initial time, compare its individual profits under both scenarios by computing its cumulative share of profits during the whole planning horizon. In this case, the channel member behaves in a farsighted manner. This channel member could also be tempted by deviating from cooperation, at any point in time during the planning horizon. The channel member computes in this situation its cumulative share of profits from the initial time to that point in time under decentralization, and compares this amount to the profit he could get if he chooses coordination from the initial time till the end of the planning horizon.

- Each channel member could adopt different reasoning where his decisions are affected by the realized profits rather than the future profits. This will correspond to a myopic behavior: the channel member's decision to stick or to deviate from the coordinated solution is the result of a comparison, under both scenarios, between its share of the cumulative profits from the initial time until any point of time during the planning horizon. By comparing both amounts, the channel member can choose between both alternatives.

To illustrate this reasoning, we consider that each channel member computes the ratio $r$ given by the following expression:

$$
r=\frac{\int_{0}^{\tilde{t}} T P(t) d t}{\int_{0}^{\tilde{t}} T P^{I}(t) d t}-1 .
$$

Because $\tilde{t}$ corresponds to the time point such that $T P(t)>T P^{I}(t)$, the ratio $r$ measures in percentage terms the increment of the total profits of the channel when applying the decentralized policy from 0 to $\tilde{t}$ with respect to the centralized policy along the same time interval. ${ }^{15}$

We compute this ratio for the following values of the model's parameters (the benchmark case):

$$
c=0, \rho=0.1, \alpha=1, \beta=1, \gamma=10, \lambda=100, R_{0}=0.3,
$$

and perform some sensitivity analysis to illustrate the impact of the parameters $\left(R_{0}, \gamma, \lambda\right)$ on the level of this ratio. The results are provided in the following table:

\footnotetext{
${ }^{15}$ Note here that this comparison is based on total channel profits rather than individual profits. For the comparison of individual profits, we introduce at the end of this section a discussion on the impact of parameter $\theta$.
} 


\begin{tabular}{c|cc} 
& $R_{0}=0.3$ & $R_{0}=0.29$ \\
\hline$\tilde{t}$ & 0.001529 & 0.00311474 \\
$r$ & $8.35 \%$ & $22.12 \%$
\end{tabular}

By comparing both results, we can conclude that a small reduction of the initial reference price from $R_{0}=0.3$ to $R_{0}=0.29$ leads to a larger interval $[0, \tilde{t})$ where the total channel profits are higher under decentralization (the length of this interval is doubled). Importantly, the percentage of the increment of the total profits obtained under decentralization vs. vertical integration along the interval $[0, \tilde{t})$ increases from $8.35 \%$ to $22.12 \%$. This important increment highlights the real incentive that the channel has to implement the decentralized policy when the game starts.

Similarly, we prove that an increase in the parameter $\gamma$ from its initial value $\gamma=10$ to $\gamma=11$ has a similar impact on the time interval $[0, \tilde{t})$ and contributes to an increase in the ratio $r$ from $8.35 \%$ to $26.54 \%$, with respect to the benchmark case.

Finally, our sensitivity analysis for the parameter $\lambda$ indicates that the impact of this parameter on the length of the time interval $[0, \tilde{t})$ is not monotonous whereas its impact on the profits ratio $r$ is negative. The following table collects the values of $\tilde{t}$ and $r$ :

\begin{tabular}{c|ccccccc} 
& $\lambda=1000$ & $\lambda=200$ & $\lambda=100$ & $\lambda=75$ & $\lambda=50$ & $\lambda=40$ & $\lambda=30$ \\
\hline$\tilde{t}$ & 0.0002201 & 0.0008968 & 0.001529 & 0.001803 & 0.001999 & 0.001836 & 0.0009771 \\
$r$ & $11.68 \%$ & $10.14 \%$ & $8.35 \%$ & $7.22 \%$ & $5.1 \%$ & $3.64 \%$ & $1.3 \%$
\end{tabular}

From this table, we note that as $\lambda$ decreases from 1000 to 50, the length of the interval $[0, \tilde{t})$ increases. We observe the opposite effect as $\lambda$ decreases from 50 to 30 . Importantly, despite the effect on $\tilde{t}$, any decrease in $\lambda$ is associated with a reduction of $r$. In other words, the smaller $\lambda$, the weaker is the incentive to implement decentralization policies at the beginning of the game. This result indicates that a shorter interval $[0, \tilde{t})$ does not always come together with a weaker incentive to play in a decentralized way. ${ }^{16}$

Let us now examine the circumstances under which each channel member could be tempted to deviate from the centralized solution.

We compute the following expressions to capture both channel members time-paths of individual profits:

$$
M P^{I}(t)=\theta T P^{I}(t), \quad R P^{I}(t)=(1-\theta) T P^{I}(t),
$$

where $T P^{I}$ denotes total profits for the whole channel under vertical integration and $M P^{I}$ and $R P^{I}$ are the manufacturer's and retailer's profits under vertical integration,

\footnotetext{
${ }^{16}$ To demonstrate the robustness of the results we conducted a similar sensitivity analysis for different levels of the model's parameters. For all of these simulations, we found that the time interval $[0, \tilde{t})$ could be multiplied by 2 (at least), whereas the ratio $r$ could be multiplied by 3 or 4 , with respect to their benchmark cases. The largest time interval where $r$ reaches the highest level is obtained for the following values of the parameters, $\gamma=11, \lambda=50$ and $R_{0}=0.3$, for which $\tilde{t}=0.0062705$ and $r=20.28 \%$.
} 
respectively, with $\theta$ as before the percentage of the total profits under vertical integration.

To analyze the incentive or disincentive that the channel members could have to deviate or not from the vertical integrated channel, we ran numerical simulations to compare the retailer's and manufacturer profits under decentralization and under vertical integration. For each scenario of the model parameters for the benchmark case, we considered the three possible values of $\theta$ represented by $\theta=0.75,0.5$ and 0.25 , denoted as the large, medium and low values respectively. These values correspond to the bounds of the intervals computed for the case where $\gamma=0$. For all the values of $\theta$, we found that there is at least one channel member that could be tempted by deviating from the cooperative solution during the time interval $[0, \tilde{t})$. Indeed, for the highest value of $\theta$, our results indicate that both the manufacturer and the retailer obtain more profits under decentralization. Thus, both of them have an incentive to end their cooperation before the channel reaches the steady state. For medium and low values of $\theta$, we found that only the manufacturer has an incentive to deviate. ${ }^{17}$

\section{Conclusion}

One of the main questions addressed in this study investigates whether the results on the efficiency of price coordination in bilateral monopolies still hold when we take into account the carry-over effects of retail prices. Indeed, we examined a dynamic model where retail price variations contribute to the building of a reference price in consumers' minds. This reference affects their current and future decisions regarding the purchase of products and thus also affects firms' subsequent pricing decisions and profits.

With this model, we proved that the results under a dynamic setting for the level of retail prices, profits and consumer surplus in coordinated and non-coordinated channels do not coincide with those obtained in the static literature. These differences are observed for some values of the initial reference price and for some periods of the planning horizon. Interestingly, we were able to characterize the initial time-period under which channel efficiency is improved under decentralization and identify different situations where channel members could be tempted to end their cooperation during this time interval. We also proved that this time horizon could be longer if the initial reference price is reduced and when the consumer-memory parameter capturing their loyalty to the brand $(\lambda)$ decreases.

The problem addressed in this study is not a simple one, especially when it is solved in a dynamic setting. Finding analytical solutions for differential games in vertical structures is a real challenge, which we met but at the cost of adopting some simplifying

\footnotetext{
${ }^{17}$ We ran additional simulations where we kept the model's parameters at their benchmark values except for $R_{0}$ then for $\gamma$. All of our results confirm that in all of the cases, there is at least one channel member that has an incentive to end the cooperation during the time interval $[0, \tilde{t})$. Remember that in the static setting (equivalent to the case where $\gamma=0$ ), we found that both channel members benefit from cooperation for $0.5 \leq \theta \leq 0.75$.
} 
assumptions and resorting to some numerical simulations to provide answers to our research questions. Indeed, we had to restrict our analysis of the issue of coordination to a unique scenario where consumers are risk neutral in their evaluations of gains and losses. Notice that this assumption does not remove the impact of the reference price in the model. It only states that the perceived gains or losses from a lower or higher retail price in accordance to the reference price are assumed to be the same. Krishnamurthi et al. (1992) observed such reasoning for situations where consumers are loyal to the brand. The choice to investigate only this scenario was made for two reasons. First, we wanted to guarantee the continuity of expression (2) and to thus keep the expressions simple enough to maintain the focus on the main objective of the study. Second, we relied on the results of Fibich et al. (2003) who studied the pricing strategies of firms under the scenarios of loss aversion. The authors proved that the optimal solution is equal to a solution of the symmetric case. Thus, asymmetry has no effect on either the optimal pricing strategies or on profits. From a qualitative perspective, our results are in line with these findings. Nevertheless, future extensions of this study should provide exact answers to these scenarios.

Furthermore, we made the choice of introducing the carry-over effects of retail prices by following Fibich et al. (2003), who consider that reference price to be based on the exponential smoothed values of past prices. Some authors working in this area use alternative ways to model the reference-price evolution. Nasiry and Popescu (2011) for example suggested the use of a peak-end rule, where the reference price is modeled as a weighted average of the last and the lowest prices paid by consumer. An extension of this study could be the modeling of the reference price as suggested by Nasiry and Popescu (2011).

Finally, it is important to mention that this study investigated the issue of channel coordination for bilateral monopolies only. Further research on this issue should explore this question for competitive channels by introducing competition at either one channel level or of both (i.e., the full competitive channel). 


\section{Appendix A}

\section{Proof of Propositions 1, 2 and 3}

The first step in a Stackelberg game is the computation of the follower's (retailer's) reaction function, that is, the retail price $(p)$ as a function of the transfer price $(w)$ and the reference price $(R)$.

When solving his optimization problem, the retailer takes into account the reference price evolution. The Hamilton-Jacobi-Bellman (HJB) equation for the retailer is

$$
\rho V^{R}(R)=\max _{p \geq 0}\left[(p-w)(\alpha-\beta p-\gamma(p-R))+\left(V^{R}\right)^{\prime}(R) \lambda(p-R)\right],
$$

where $V^{R}(R)$ denotes the retailer's value function.

From the first-order optimality conditions we get the retailer's reaction function in (6).

We now compute the expression of the manufacturer's optimal pricing and value function, $V^{M}(R)$.

The sufficient condition for a stationary feedback Stackelberg equilibrium requires us to find a bounded and continuously differentiable function, denoted by $V^{M}(R)$, which satisfies, for all $R(t) \geq 0$, the HJB equation for the manufacturer where the retailer's reaction function given in (6) has been replaced. We first concentrate on finding solutions for the HJB equation. This equation is given by

$$
\rho V^{M}(R)=\max _{w \geq 0}\left[(w-c)(\alpha-\beta p-\gamma(p-R))+\left(V^{M}\right)^{\prime}(R) \lambda(p-R)\right],
$$

where $p$ has to be replaced by the retailer's reaction function given in (6).

The first-order optimality condition reads

$$
w(R)=\frac{\alpha+\gamma R+c(\beta+\gamma)+\lambda\left(\left(V^{M}\right)^{\prime}(R)-\left(V^{R}\right)^{\prime}(R)\right)}{2(\beta+\gamma)},
$$

if this expression is positive, and zero otherwise.

The optimal retail price is obtained by replacing the above expression of the wholesale price (24) in the retailer's reaction function (6):

$$
p(R)=\frac{3(\alpha+\gamma R)+c(\beta+\gamma)+\lambda\left(\left(V^{M}\right)^{\prime}(R)+\left(V^{R}\right)^{\prime}(R)\right)}{4(\beta+\gamma)},
$$

if this expression is positive, and zero otherwise.

Guided by the model's linear-quadratic structure, we assume that the manufacturer's and the retailer's value functions are quadratic and given by (7) and (8).

Taking into account this specification for the manufacturer's and the retailer's value functions in (24) and (25), after an easy computation, the optimal pricing strategies 
can be written as

$$
\begin{aligned}
& w(R)=\frac{\alpha+\gamma R+c(\beta+\gamma)+\lambda\left(M_{1} R+M_{2}-N_{1} R-N_{2}\right)}{2(\beta+\gamma)}, \\
& p(R)=\frac{3(\alpha+\gamma R)+c(\beta+\gamma)+\lambda\left(M_{1} R+M_{2}+N_{1} R+N_{2}\right)}{4(\beta+\gamma)},
\end{aligned}
$$

if there are positive expressions, and zero otherwise.

Inserting (24) and (25) into (23), the six Riccati equations that characterize the coefficients of the value functions $M_{i}, N_{i}, i=1,2,3$ are determined by identification.

The coefficients of the retailer's value function are linked to the coefficients of the manufacturer's value function as follows:

$$
N_{1}=\frac{M_{1}}{2}, \quad N_{2}=\frac{M_{2}}{2}, \quad N_{3}=\frac{M_{3}}{2} .
$$

The coefficients of manufacturer's value function, $M_{1}, M_{2}$ and $M_{3}$, are:

$$
\begin{aligned}
& M_{1}=2 \frac{4 \beta(2 \lambda+\rho)+\gamma(3 \lambda+4 \rho) \pm \sqrt{(4 \beta(2 \lambda+\rho)+\gamma(3 \lambda+4 \rho))^{2}-9 \gamma^{2} \lambda^{2}}}{9 \lambda^{2}}, \\
& M_{2}=2 \frac{\alpha\left(2 \gamma+5 M_{1} \lambda\right)-c(\beta+\gamma)\left(2 \gamma-3 M_{1} \lambda\right)}{3 \lambda\left(2 \gamma-3 M_{1} \lambda\right)+16(\gamma \rho+\beta(\lambda+\rho))}, \\
& M_{3}=\frac{4(\alpha-c(\beta+\gamma))^{2}+M_{2} \lambda\left(12 c(\beta+\gamma)+9 M_{2} \lambda+20 \alpha\right)}{32(\beta+\gamma) \rho} .
\end{aligned}
$$

A sufficient condition guaranteeing that the expressions in (7) and (8) are the manufacturer's and the retailer's value functions and that (24) and (25) are the pricing strategies is given by

$$
\lim _{t \rightarrow \infty} e^{-\rho t} V^{M}(R(t))=0, \quad \lim _{t \rightarrow \infty} e^{-\rho t} V^{R}(R(t))=0,
$$

where $R(t)$ is the solution of the closed-loop dynamics obtained after substituting the optimal pricing strategies (24) and (25) into the reference price dynamics given by (1).

This solution can be written as in (17), where the steady state of the reference price denoted by $R_{s s}$ is given by (12). The steady state is globally stable if and only if

$$
8 \beta+2 \gamma-3 M_{1} \lambda>0,
$$

and is positive if and only if the following condition on the parameters is satisfied:

$$
6 \alpha+2 c(\beta+\gamma)+3 M_{2} \lambda>0 .
$$

Both expressions of $M_{1}$ are positive. The stability condition for the steady state is only satisfied if the expression of $M_{1}$ with the square root affected by the negative sign is chosen. If the other expression is chosen, the stability condition is never satisfied. The positivity condition of the steady state is always satisfied.

The quadratic functional specifications in (7) and (8) allow conditions in (26) to be satisfied whenever the reference price is bounded. This condition is guaranteed because the steady state is globally asymptotically stable. 


\section{Appendix B}

\section{Proof of Propositions 4 and 5}

Following the same steps as in Appendix A, the optimal retail price for the vertically integrated channel in (13) can be derived from the following HJB equation:

$$
\rho V^{I}(R)=\max _{p \geq 0}\left[(p-c)(\alpha-\beta p-\gamma(p-R))+\left(V^{I}\right)^{\prime}(R) \lambda(p-R)\right],
$$

where $V^{I}(R)$ denotes the value function of the vertically integrated channel.

The coefficients of the value function for the vertically integrated channel, $I_{1}, I_{2}$ and $I_{3}$, can be obtained by identification:

$$
\begin{aligned}
& I_{1}=\frac{\gamma(\lambda+\rho)+\beta(2 \lambda+\rho) \pm \sqrt{(\beta+\gamma)(2 \lambda+\lambda)(\gamma \rho+\beta(2 \lambda+\rho))}}{\lambda^{2}} \\
& I_{2}=\frac{\alpha\left(\gamma+I_{1} \lambda\right)-c(\beta+\gamma)\left(\gamma-I_{1} \lambda\right)}{2 \beta(\lambda+\rho)+\gamma(\lambda+2 \rho)-I_{1} \lambda^{2}} \\
& I_{3}=\frac{(\alpha-c(\beta+\gamma))^{2}+I_{2} \lambda\left(I_{2} \lambda+2 c(\beta+\gamma)+2 \alpha\right)}{4(\beta+\gamma) \rho}
\end{aligned}
$$

The optimal time-path of the reference price can be written as in (18), where $R_{s s}^{I}$ refers to the steady state of the reference price and is given by (14). The steady state is globally stable if and only if

$$
2 \beta+\gamma-I_{1} \lambda>0
$$

and is positive if and only if

$$
\alpha+c(\beta+\gamma)+I_{2} \lambda>0
$$

Both expressions of $I_{1}$ are positive. The stability condition for the steady state is only satisfied if the expression of $I_{1}$ with the square root affected by the negative sign is chosen. If the other expression is chosen, the stability condition is never satisfied. The positivity condition of the steady state is always satisfied. 


\section{References}

[1] Benchekroun, H., G. Martín-Herrán, and S. Taboubi (2009). Could Myopic Pricing Be a Strategic Choice in Marketing Channels? A Game Theoretic Analysis. Journal of Economic Dynamics and Control 33 (9), 1699-1718.

[2] Dean, J. (1969) Pricing Pioneering Products. The Journal of Industrial Economics $17,165-179$.

[3] Dodds, W.B., K.B. Monroe, and D. Grewal (1991). Effects of Price, Brand, and Store Information on Buyers' Product Evaluations. Journal of Marketing Research 28 (3), 307-319.

[4] Fibich, G., A. Gavious, and O. Lowengart (2003). Explicit Solutions of Optimization Models and Differential Games with Nonsmooth (Asymmetric) ReferencePrice Effects. Operations Research 51, 721-734.

[5] Fibich, G., A. Gavious, and O. Lowengart (2007). Optimal Price Promotion in the Presence of Asymmetric Reference-Price Effects. Managerial and Decision Economics 28, 569-577.

[6] Greenleaf, E.A. (1995). The Impact of Reference Price Effects on the Profitability of Price Promotions. Marketing Science 14, 82-104.

[7] Helson, H. (1964). Adaptation-Level Theory, New York: Harper \& Row.

[8] Ingene, C.A., S. Taboubi, and G. Zaccour, (2012). Game-Theoretic Coordination Mechanisms in Marketing Channels: Integration and Extensions for Models Without Competition. Journal of Retailing 88 (4), 476-496.

[9] Jeuland, A.P., and S.M. Shugan (1983). Managing Channel Profits. Marketing Science 2 (3), 239-272.

[10] Kalyanaram, G., and R.S.Winer (1995). Empirical Generalizations from Reference Price Research. Marketing Science 14 (3), G161-G169.

[11] Kahneman, D., and A. Tversky (1979). Prospect Theory: An Analysis of Decision Making under Risk. Econometrica 47, 263-291.

[12] Kopalle, P.K., and R.S. Winer (1996). A Dynamic Model of Reference Price and Expected Quality. Marketing Letters 7, 41-52.

[13] Kopalle, P.K., A.G. Rao, and J.L. Assunçao (1996). Asymmetric Reference Price Effects and Dynamic Pricing Policies. Marketing Science 15 (1), 60-85.

[14] Krishnamurthi, L., T. Mazumdar and S.P. Raj (1992). Asymmetric Response to Price in Consumer Brand Choice and Purchase Quantity Decisions. Journal of Consumer Research 19, 387-400. 
[15] Martín-Herrán, G., S. Taboubi, and G. Zaccour (2012). Dual Role of Price and Myopia in a Marketing Channel. European Journal of Operational Research 219 (2), 284-295.

[16] Mazumdar, T., S.P. Raj, and I., Sinha (2005). Reference Price Research: Review and Propositions. Journal of Marketing 69, 84-102.

[17] Monroe, K.B. (1979). Pricing: Making Profitable Decisions, New York, NY: McGraw-Hill.

[18] Nasiry, J., and I. Popescu (2011). Dynamic Pricing with Loss-Averse Consumers and Peak-End Anchoring. Operations Research 59 (6), 1361-1368.

[19] Nerlove, M., and K.J. Arrow (1962). Optimal Advertising Policy Under Dynamic Conditions, Economica. 29, 129-142.

[20] Popescu, I., and Y. Wu (2007). Dynamic Pricing Strategies with Reference Effects. Operations Research 55 (3), 413-429.

[21] Sorger, G. (1988). Reference Price Formation and Optimal Pricing Strategies. In: Optimal Control Theory and Economic Analysis 3, G. Feichtinger (ed.), Elseveir, Amsterdam, 97-120.

[22] Thaler, R. (1985). Mental Accounting and Consumer Choice. Marketing Science $4(3), 199-214$.

[23] Völckner, F., (2008). The Dual Role of Price: Decomposing Consumers' Reactions to Price. Journal of the Academy of Marketing Science 36, 359-377.

[24] Weisstein, F.L., K.B. Monroe and M. Kukar-Kinney (2013). Effects of Price Framing on Consumers' Perceptions of Online Dynamic Pricing Practices. Journal of the Academy of Marketing Science 41, 501-514.

[25] Zaccour, G. (2006). On the Coordination of Dynamic Marketing Channels and Two-Part Tariffs. Automatica 44 (May), 1233-1239. 